\title{
Mental Health, Work Engagement and Meaningful Work-Role Fit of Industrial Psychologists: A Latent Profile Analysis
}

\author{
Llewellyn Ellardus van $\mathrm{Zyl}^{1,2}$ (1) $\cdot$ Sebastiaan Rothmann ${ }^{2} \cdot$ Carmine Nieman $^{2}$
}

Received: 4 December 2018/Accepted: 14 October 2019/Published online: 30 March 2020

(C) The Author(s) 2020

\begin{abstract}
The purpose of this study was to identify distinctive mental health profiles for industrial psychologists based on the Mental Health Continuum. Further, it aimed to determine how these profiles differ with respect to workrole fit, meaningfulness and work engagement. It also aimed to investigate whether industrial psychologists within managerial or specialist differ in respect of different types of mental health. An online cross-sectional survey design was employed to draw a census sample $(n=274)$ from all South African industrial psychologists. A biographical questionnaire, the Work-Role Fit Scale, the Psychological Meaningfulness Scale, the Work Engagement Scale, and the Mental Health Continuum-Short Form were administered. Descriptive statistics, correlations, latent profile analysis, MANOVAs and ANOVAs were computed. Three mental health profiles for industrial psychologists were identified: languishing, moderately mentally healthy and flourishing. Significant differences between the three mental health profiles and experiences of meaningful work-role fit and work engagement were found, but not between experiences of managerial roles. The results show that individuals with different mental health profiles, experience work and its related outcomes, differently. Therefore, in order to enhance meaningful work-role fit and work engagement of industrial psychologists, a one-size-fits-all model may not be appropriate.
\end{abstract}

Llewellyn Ellardus van Zyl

llewellyn101@gmail.com

1 Department of Human Performance Management, University of Eindhoven, Eindhoven, The Netherlands

2 Optentia Research Focus Area, North-West University (VTC), Vanderbijlpark, South Africa
Keywords Mental health · Meaningful work-role fit . Work engagement · Industrial psychologists . Managerial roles

\section{Introduction}

Industrial psychologists are predisposed to develop mental health problems due to the unique physical, emotional and psychological demands inherent to their profession (Meyers, 2007; Jorgensen \& van Zyl, in press; Schaerer, 2011). These demands stem from taking personal responsibility for the wellbeing of clients, balancing the needs of the organisation with those of employees, working extended hours with limited time to recover, facing challenging working conditions and having to manage the ever-decreasing availability of job-and personal resources (Barkhuizen, Jorgensen, \& Brink, 2015; Khamisa, Peltzer, Ilic, \& Odenburg, 2016, 2017; Ogińska-Bulik, 2006). Demands are compounded by a collectively shared identity crisis which stems from an inability to distinguish their value proposition from traditional human resource management (Coetzee \& Van Zyl, 2014; Van Zyl, Nel, Stander, \& Rothmann, 2016) and the clinical psychology discipline (Schaerer, 2011). Industrial psychologists therefore spend a vast majority of their time on activities unrelated to their area of expertise which results in a significant discrepancy between the activities which provide them with energy and those that distract from such (Van Zyl, Deacon, \& Rothmann, 2010). This misfit between the person and work, coupled with the collectively shared identity crisis and the unique demands inherent to their profession, may result in increased experiences of meaninglessness and disengagement (Barkhuizen et al., 2015; McCann et al., 2013) which 
in turn has negative consequences for their overall mental health (Van Zyl et al., 2010).

Psychological problems not only affect the mental health of industrial psychologists, but may have various negative implications for both the client (e.g. providing poor quality service) and/or the organisation (e.g. decreased performance, high-staff turnover, increased legal proceedings associated with negligence) in which the he/ she functions (Lim, Kim, Kim, Yang, \& Lee, 2010; Lomas, Medina, Ivtzan, Rupprecht, \& Eiroa-Orosa 2018; Veage et al., 2014). Since industrial psychologists play a vital role in the wellbeing of employees, and that they usually occupy strategic functions at an organisational level, their level of mental (ill)health could spill over to other employees (Meyers, 2007) and result in poor decisionmaking which inevitably could steer the organisation down the proverbial "wrong path". Given the important role which these individuals play at work, it is surprising that research into the mental health of industrial psychologists is relatively non-existent (Van Zyl et al., 2010, 2016).

Although a minute amount of academic interest is centred around the mental health of their clinical psychological counterparts (c.f. Cushway \& Tyler, 1996; Di Benedetto \& Swadling, 2014), no studies on the mental health of industrial psychologists could be found within the literature. It is therefore not clear how the mental health of industrial psychologists should be conceptualised, measured or managed. It is also not clear how mentally healthy industrial psychologist are, nor what the specific factors contribute to such. Resultantly, how industrial psychologist presenting with different levels of mental health differ with respect to their experience of these attributing factors is also not evident. In order to limit the potential negative impact of the industrial psychologist's mental (ill) health on various stakeholders, and to retain skilled practitioners within the profession, a greater understanding of these issues are required.

\section{Mental Health}

The World Health Organisation (2014) defined mental health as a complete "state of well-being in which every individual realizes his or her own potential, can cope with the normal stresses of life, can work productively and fruitfully, and is able to make a contribution to her or his community". From this perspective, the positive dimensions of mental health are emphasised, where wellbeing is a function of optimal development and growth rather than the absence of infirmity, disease or psychopathology (Peterson \& Seligman, 2004; WTO, 2014). Framed differently, mental health is seen as a state of complete being where one is free from psychopathology and where one feels good, functions well and fits into with one's social context (Keyes, 2002).

This positive approach towards mental health was conceptualised by Keyes (2002) as the "Mental Health Continuum". He argued that mental health ranges on a continuum and is a function of a dynamic, yet balanced, interplay between emotional-(i.e. balanced experiences of positive and negative emotions which culminates in high levels of life satisfaction), psychological-(i.e. a feeling that one is thriving in one's personal life and that one can actualise one's potential) and social wellbeing (i.e. feelings of social integration, social contribution, social coherence, social actualisation and social acceptance as a member of a larger society) (Keyes, 2002; Rothmann, Van Zyl, \& Rautenbach, 2019). Each type of wellbeing comprises a unique set of symptoms (c.f. Table 1).

Through various combinations of the symptoms, Keyes (2005) argued that an individual's level of mental health can range on a continued spectrum from: (a) Languishing (i.e. low levels of wellbeing characterised by low or no emotional, psychological and social wellbeing), to (b) Moderate Mental Health (i.e. moderate levels of emotional, psychological and social wellbeing), to (c) Flourishing (i.e. high levels of life satisfaction/affect balance, psychological and social wellbeing). Specifically, Keyes (2007) indicated that for an individual to be classified into the languishing profile, he/she needs to report low on at least seven out of the 14 symptoms mentioned in Table 1; where 1 should be from emotional wellbeing. Similarly, for an individual to be categorised as into the flourishing profile, he needs to report high on at least seven of the symptoms; one of which again needs to be from the emotional wellbeing cluster (Keyes, 2007). If an individual does not fall into one of these two categories, they are categorised into the moderate mental health profile (Keyes, 2007).

Keyes's (2002) model provides an interesting diagnostic framework for the classification of industrial psychologists' mental health. This framework not only provides a means through which to assess various levels of wellbeing but provides structured guidelines and a holistic classification framework to classify industrial psychologists into three internationally accepted categories for mental health. It further provides a framework through which to determine the most appropriate attributing factors for the mental health of industrial psychologists. This in turn provides a means through which to empirically investigate how languishing, moderately mentally healthy and flourishing industrial psychologists differ with respect to their experience of these attributing factors.

Only four studies within the literature have specifically eluded to the attributing factors to the mental health of industrial psychologists (c.f. Barnard \& Fourie, 2007; Hitge \& Van Schalkwyk, 2018; Van Zyl et al., 2010, 2016). 
Table 1 Keyes's (2002) mental health symptomology

\begin{tabular}{|c|c|}
\hline Type of wellbeing & Symptoms \\
\hline \multirow[t]{3}{*}{ Emotional wellbeing } & 1. Happiness \\
\hline & 2. Interested in life \\
\hline & 3. Satisfied with life \\
\hline \multirow[t]{6}{*}{ Psychological wellbeing } & 1. Self-acceptance \\
\hline & 2. Environmental mastery \\
\hline & 3. Positive relations with others \\
\hline & 4. Personal growth \\
\hline & 5. Autonomy \\
\hline & 6. Purpose in life \\
\hline \multirow[t]{5}{*}{ Social wellbeing } & 1. Social contribution \\
\hline & 2. Social integration \\
\hline & 3. Social actualization \\
\hline & 4. Social acceptance \\
\hline & 5. Social coherence \\
\hline
\end{tabular}

These studies suggested that four factors could be significant contributors to and indicators of the mental health of industrial psychologists: (a) work-role fit, (b) meaningfulness, (c) work engagement and (d) whether an individual occupies a managerial or specialist function. Consequently, industrial psychologists with different mental health profiles may also differ with respect to their experiences of these factors, as well as the extent towards which these relate.

\section{Work-Role Fit and Mental Health}

Work-role fit was found to be an important predictor of various components associated with the mental health of industrial psychologists (Van Zyl et al., 2010). Work-role fit is defined as the perceived match between an individuals' strengths, personality and work preferences with the work-related roles which they occupy (May, Gilson, \& Harter, 2004). May et al. (2004) describe work-role fit as the role that an individual assumes within an organisation that allows them to be self-expressive and creative and not just goal-oriented. Research suggests that individuals will actively seek out work roles through which their "true selves" could be expressed in order to craft meaningful and engaging work (May et al., 2004; Olivier \& Rothmann, 2007).

As such, when there is perceived fit between an industrial psychologist's character (i.e. strengths, personality, skills, competencies and/or work preferences), and the role occupied within an organisation, it could lead to increased mental health (Van Zyl et al., 2010). In contrast, if industrial psychologists experience a misalignment between their work preferences, strengths or interests and their work-role requirements, they could experience lower levels of mental health (Van Zyl et al., 2010). Industrial psychologists have reported to be trapped between the operational roles of traditional human resource management and the more dynamic functions of applied psychology at work (Barnard \& Fourie, 2007, Van Zyl et al., 2010). They often spend more time on administrative-and human resourcerelated tasks, than on preferred tasks such as the counselling, coaching and the development of people (Van Zyl et al., 2010). This discrepancy could negatively influence their perceptions of fit in their jobs as well as the meaningfulness of their work which may result in lower levels of work engagement (Meyers, 2007; Van Zyl et al., 2010), and negatively affect their mental health (Seligman, 2008).

Similarly, there may also be differences between industrial psychologists presenting with different mental health profiles and work-role fit. Flourishing industrial psychologists may experience higher levels of work-role fit than languishers. This may be because a relationship between individuals' work-roles and their true selves supports their mental wellbeing (Glazer, Kozusznik, Meyers, \& Ganai, 2014). Similarly, flourishing industrial psychologists may have a large overlap between the types of activities they engage in at work and their skills, competencies, desires and interests (Van Zyl et al., 2016; Jorgensen-Graupner, \& Van Zyl 2019). Work-role misfit could, in contrast, lead to languishing, because work-role misfit is associated with frustration, pessimism and negativity (Meyers, 2007). However, given that no studies relating to the differences in mental health and work-role fit are available, it is not clear what the differences for industrial psychologists will be. 


\section{Meaningfulness and Mental Health}

Meaningfulness was found to be another important attributor to the mental health and wellbeing of industrial psychologists (Van Zyl et al., 2010). Vogt, Jenny, and Bauer (2013) argued that meaningfulness is described as the extent to which one's work is seen as worthy of commitment and involvement. Specifically, Kahn (1990) defines psychological meaningfulness as feeling that a person is receiving a proverbial investment return on the physical, cognitive or emotional energy which they invest in and at work. From this perspective, individuals subjectively judge the value of work-related tasks and goals in relation to their own ideals, values and norms (May et al., 2004). If tasks are deemed to be aligned to one's personal mission and vision, coupled with feelings that one is contributing to something larger than oneself and that one is making a "difference", then individuals will experience high levels of psychological meaningfulness (Hitge \& Van Schalkwyk, 2018; Olivier \& Rothmann, 2007).

In their study, Van Zyl et al. (2010) found that most of the industrial psychologists regarded work as a calling and experienced high level of meaningfulness. It was argued that meaningfulness could buffer against the demands and inherent occupational stressors associated with their profession (Glazer, Kozusznik, Meyers, \& Ganai, 2014). Meaningfulness further aids in the enhancement and maintenance of general mental health, life satisfaction and work engagement (Glazer et al., 2014; Olivier \& Rothmann, 2007). Similar to work-role fit, no studies relating to the differences in mental health and meaningfulness are available. Therefore, it is not clear how the level of meaningfulness will differ between languishers, the moderately mentally health and flourishers.

\section{Work Engagement and Mental Health}

As an outcome of both work-role fit and meaningfulness, work engagement (i.e. the extent towards which individuals attach and lose themselves in their work roles) is seen as another important indicator of the mental health of industrial psychologists (Van Zyl et al., 2010). May et al. (2004) pointed out that work engagement comprises a physical(i.e. physical involvement during a work task), a cognitive(i.e. cognitive alertness during a work task) and an emotional component (referring to the emotional connectedness to a work task). When individuals report high levels of emotional, cognitive and physical engrossment in their work, they are more inclined to flourish, where lower levels of such are usually associated with languishing (ArizaMontes, Molina-Sanchez, Ramirez-Sobrino, \& Giorgi, 2018).
Industrial psychologists have shown to present aboveaverage levels of work engagement (Van Zyl et al., 2010), which could positively affect their mental health. Research has shown that industrial psychologists are inclined to use their external resources, prepare themselves for selfchange, apply their knowledge and display a preference to progress in all life domains such as studies, career and personal life; thus, making use of their opportunities to apply their strengths to engage in work (De Jager-van Straaten, Jorgensen, Hill, \& Nel, 2016; Peterson \& Seligman, 2004). However, Van Zyl et al. (2010) argued that work engagement and the association with mental health need further investigation. From the available literature, it is also not clear how industrial psychologists with different levels of mental health would differ in respect of their level of work engagement.

\section{Managerial Versus Specialist Roles and Mental Health}

As indicated in the arguments above, the alignment between the self and a work role is an important contributor to mental health. Although industrial psychologists occupy various roles and functions at work, most of these could be considered an industrial psychological specialist role or a human resource function (Barnard \& Fourie, 2007; Van Zyl et al., 2016). However, Van Zyl et al. (2010) found that a large portion of their sample reported to be in managerial positions; a role which is neither an inherent part of an industrial psychologist's training nor a fundamental part of his specialist skill set. A managerial role is characterised by having responsibility and authority to manage the workrelated functioning of employees (Petrou, Van den Heuvel, \& Schaufeli, 2017). Non-managerial roles can be defined as specialist roles that require particular experience, expert knowledge and skills (Field et al., 2018). If specialists (with a preference for specialised tasks, procedures and functions) are promoted into managerial positions, it could negatively affect their mental health (Charan, Drotter, \& Noel, 2010; Drotter, 2011). Drotter (2011) argues that this is because the core functions of a leadership/management position differ significantly from those of specialist functions. Particularly, when an individual does not show a preference for managing people lacks leadership experience or lacks the capabilities needed to effectively manage people, it leads to increased levels of frustration, psychological distress, anxiety and job dissatisfaction (Charan et al., 2010; Ruderman \& Ohlott, 1994).

The roles industrial psychologists fulfil within organisations can be aggregated to either a specialist or a managerial function. Industrial psychologists are trained as behavioural specialists that develop, and apply psychological theories, processes, methods and paradigms to 
optimise individual, group, organisational and societal potential (Grand et al., 2018; Landy, \& Conte, 2016; Van Zyl et al., 2016). Research shows that the majority of industrial psychologist occupy specialist roles which require specific experience and expert knowledge (Barnard \& Fourie, 2007; Landy, \& Conte, 2016). Further, Van Zyl et al. (2010) found that industrial psychologists even show a preference for these specialist psychological functions. Although industrial psychologists are exposed to leadership assessment and development frameworks as part of their formal academic training, limited focus is placed on equipping them with the necessary skills, and abilities needed to be effective managers or leaders. Given the preference for specialist functions, and the lack of preparation for leadership positions during their formal training, industrial psychologists in managerial positions may be more likely to languish than to flourish.

\section{Determining the Mental Health Profiles: Latent Profile Analysis}

Although Keyes (2007) provides a diagnostic "count-based framework" for the classification of languishers, moderates and flourishers, no study was found that empirically confirms the manifestation of these profiles within any population. Although there are sound theoretical arguments as to how the three profiles should and could be estimated (Keyes, 2002, 2007), reducing the factor structure to languishing, moderately mentally healthy and flourishing with a confirmatory factor analytic approach, poses various analytical challenges (e.g. results in poor model fit, negative residual variances and non-positive definite errors) (Masyn, 2013). An exploratory, non-variable-centric approach is required to empirically investigate the presence of different mental health profiles. Oberski (2016) suggested the use of latent profile analysis (LPA) in order to determine if different types of (mental health) profiles, in samples where similar patterns amongst variables could meaningfully be extracted. LPA is a type of person-centred analysis that aims to determine whether homogeneous subgroups of individuals within a dataset can be distinguished by similar clusters in responses, on similar observed continuous variables (i.e. measured items), by similar groups of individuals (Berlin, Williams, \& Parra, 2014). In other words, it aims to identify homogenous subgroups of individuals that pose similar or unique attributes, which is practically different from other subgroups (Berlin et al., 2014; Oberski, 2016). It does so in much the same way as in traditional factor analytic approaches; however, instead of item-to-factor extractions, the focus is on determining patterns of shared variance. Patterns of shared variance amongst industrial psychologists on the three different components of mental health can be extracted into meaningful profiles; which in return are heuristically evaluated in order to determine the exact amount of profile that are evident within the given data (Berlin et al., 2014). The focus in this type of analysis therefore shifts focus from factors (derived from a factor analysis), into complex and meaningful patterns variance that are evident amongst people (Stanley, Kellermanns, \& Zellweger, 2017).

This approach is more beneficial than traditional variable-centric approaches (TVCA), as it offers an empirically parsimonious means through which to classify industrial psychologists' mental health using a theoretically meaningful and established framework (Marsh et al., 2009). TVCAs used to determine subgroups have various limitations which LPA effortlessly addresses. ${ }^{1}$ First, TVCAs (such as traditional exploratory factor analyses) are limited in the extent towards which they are able to detect complex interactions, amongst multiple variables (Aguinis, Gottfredson, \& Wright, 2011; Meyer, Stanley, \& Vandenberg, 2013). Given the magnitude of factors influencing mental health, and the shared variance between emotional, psychological and social wellbeing, employing a TVCA to accurately classify mental health profiles presents various challenges which is not viable within the current study. Second, TVCAs assume that samples are naturally homogeneous and therefore that the given the latent mean structures of factors being measured to estimate relationships amongst variables are also homogenous (Berlin et al., 2014; Oberski, 2016). For example, employing a confirmatory factor analytical approach to confirm a factor structure of an instrument within a diverse sample of individuals from various cultural backgrounds assumes that the model is exactly the same for all subgroups or subpopulations within the sample (which we know from crosscultural psychology is not the case). Third, TVCAs assume that independent latent variables have separate effects on dependent variables. LPA on the other hand assumes that patterns within variables and between variables exists and considers such jointly (Geiser, 2012). Finally, TVCAs employ subjective criteria (such as "eye balling", HARKing or "(mis)quoting theory to justify claims") rather than objective criteria to estimate differences and may therefore be prone to bias (Geiser, 2012). Therefore, given the nature of mental health, the use of LPA to extract different profiles is justified.

\section{The Current Study}

The purpose of this current study was to identify distinctive mental health profiles for industrial psychologists, based on

\footnotetext{
${ }^{1}$ For a full comparison between LPA and TVCAs, please refer to Table 1 in Meyer et al. (2013).
} 
Keyes' (2002) mental health framework, and to determine how these profiles differ in respect of work-role fit, meaningfulness and work engagement. Further it aimed to determine whether managers and specialists differ in respect of the mental health profiles.

Stemming from this primary aim, the following specific objectives will be investigated:

1. To determine the types of mental health profiles of industrial psychologists

2. To study the differences between work-role fit, meaningfulness, work engagement and the mental health profiles of industrial psychologists

3. To investigate the differences between managers and specialist in respect of the mental health profiles of industrial psychologists

\section{Method}

\section{Participants and Sampling Strategy}

This study forms part of a research project relating to the mental health of industrial and organisational psychologists in South Africa. Data were collected in 2014 through employing a census-based sample from the entire population of industrial psychologists in South Africa. Census sampling refers to the entire population being studied (Molenberghs, 2010). The sample comprises 274 registered industrial psychologists from the total population $(N=1167)$. Table 2 indicates that most of the participants were Afrikaans (56.3\%), White (83.5\%), Afrikaans speaking $(56.3 \%)$ females $(63.6 \%)$ between the ages of 41 and $50(29.8 \%)$. The majority of the participants were registered as industrial psychologists between 0 and 5 years $(42.6 \%)$ and were employed in specialist positions $(52.9 \%)$ by a large organisation $(71.7 \%)$.

\section{Measuring Battery}

The following instruments were employed as part of this study:

A biographical questionnaire to gather information pertaining to gender, age, native language, ethnicity, years registered as an industrial psychologist, managerial and specialist roles and type of employer.

The Mental Health Continuum-Short Form (MHC-SF) was employed to measure the mental health of South African industrial psychologists (Keyes, 2002). This scale measures mental health via the three types of wellbeing with a total of 14 items, averaging from 1 (Never) to 5 (All of the time). Three items measure emotional wellbeing (e.g. "During the past month, how often did you feel happy?"; six items measure psychological wellbeing (e.g. "How often during the past month did you feel that you liked most parts of your personality?"); and five items measure social wellbeing (e.g. "How often during the past month did you feel that you belonged to a community?"). The reliability of the MHC-SF was confirmed $(\alpha=0.80)$ (Keyes, 2002).

The Work-Role Fit Scale (WRFS) (May et al., 2004) was employed to measure the fit between individuals' jobs and their self-concepts. Work-role fit was measured by four items on a five-point Likert scale varying from: 1 (totally agree) to 5 (totally disagree). An example item is: "My job 'fits' how I see myself". The reliability of the Work-Role Fit Scale was previously confirmed $(a=0.90)$ (Olivier \& Rothmann, 2007).

The Psychological Meaningfulness Scale (PMS) was employed to measure the psychological meaningfulness of industrial psychologists (May et al., 2004). This scale measures the extent towards which an individual believes he/she is receiving a return on investment on the energy they invest at work. It is measured by six items on a fivepoint Likert scale averaging from 1 (never) to 5 (always). Examples of items included are: "The work I do on this job is very important to me" and "My job activities are personally meaningful to me". The reliability of the Psychological Meaningfulness Scale was previously confirmed ( $\alpha=0.90$ ) (May et al., 2004).

The Work Engagement Scale (WES) (May et al., 2004) was adapted and employed to measure the work engagement of South African industrial psychologists This instrument consists of 17 items that measure work engagement with a Likert-type scale varying from 1 (strongly disagree) to 5 (strongly agree). This scale reflects the three work engagement components from Kahn's (1990) definition, namely cognitive ("When I am working, I often lose track of time"), emotional ("I often feel emotionally attached to my job") and physical engagement ("I am full of energy in my work") (Van Zyl et al., 2010). The reliability of the WES was confirmed with a one-factor engagement model $(\alpha=0.72)$ (Olivier $\&$ Rothmann, 2007).

\section{Procedure}

The study was presented at the Society for Industrial and Organisational Psychology of South Africa's (SIOPSA) executive committee meeting on April 2013, in order to obtain permission to conduct the research and to gain access to their database of industrial psychologists. After that participant information was gained through purchasing a list of information of all registered industrial psychologists within South Africa from the HPCSA. The HPCSA's list was compared with SIOPSA's database to ensure that all industrial psychologists were targeted An online 
Table 2 Personal characteristics of participants $(N=274)$

\begin{tabular}{|c|c|c|c|}
\hline Item & Category & Frequency & Percentage \\
\hline \multirow[t]{2}{*}{ Gender } & Male & 99 & 36.4 \\
\hline & Female & 173 & 63.6 \\
\hline \multirow[t]{10}{*}{ Native language } & Afrikaans & 153 & 56.30 \\
\hline & English & 102 & 37.5 \\
\hline & Sepedi & 2 & 0.7 \\
\hline & Zulu & 3 & 1.1 \\
\hline & Sesotho & 2 & 0.7 \\
\hline & Setswana & 2 & 0.7 \\
\hline & IsiSwati & 1 & 0.4 \\
\hline & IsiTsonga & 3 & 1.1 \\
\hline & IsiXhosa & 3 & 1.1 \\
\hline & Other & 1 & 0.4 \\
\hline \multirow[t]{5}{*}{ Age } & 22 to 30 Years & 54 & 19.9 \\
\hline & 31 to 40 Years & 72 & 26.5 \\
\hline & 41 to 50 Years & 81 & 29.8 \\
\hline & 51 to 60 Years & 46 & 16.9 \\
\hline & 61 Years and above & 19 & 7.0 \\
\hline \multirow[t]{4}{*}{ Ethnicity } & White & 227 & 83.5 \\
\hline & African & 18 & 6.61 \\
\hline & Coloured & 11 & 4.04 \\
\hline & Indian & 16 & 5.88 \\
\hline \multirow[t]{7}{*}{ Years registered } & 0 to 5 Years & 116 & 42.6 \\
\hline & 6 to 10 Years & 52 & 19.1 \\
\hline & 11 to 15 Years & 37 & 13.6 \\
\hline & 16 to 20 Years & 28 & 10.3 \\
\hline & 21 to 25 Years & 16 & 5.9 \\
\hline & 26 to 30 Years & 10 & 3.7 \\
\hline & 31 Years and above & 13 & 4.8 \\
\hline \multirow[t]{5}{*}{ Organisation size } & Micro-business & 55 & 20.2 \\
\hline & Small business & 19 & 7 \\
\hline & Medium business & 17 & 6.3 \\
\hline & Large business & 175 & 64.3 \\
\hline & NGO & 6 & 2.2 \\
\hline \multirow[t]{2}{*}{ Manager } & Managerial position & 128 & 47.1 \\
\hline & Specialist position & 144 & 52.9 \\
\hline
\end{tabular}

questionnaire was constructed and sent out via email on November 2013 to all the South African industrial psychologists registered with the HPCSA. The questionnaire contained a cover letter, explaining all the ethical considerations (confidentiality, anonymity, informed consent and the right to withdraw) as well as an explanation of the research procedure. The data were automatically captured upon completion of the surveys on a secured SSL server. Data were securely downloaded in SPSS format, and cleaned in order to be processed.

\section{Statistical Analysis}

Statistical analysis was conducted with SPSS 24.0 (SPSS, 2018) and Mplus 8.1 (Muthén \& Muthén, 1998-2018). First, to determine the presence of common method bias Harman's (1976) single factor test (Tehseen, Ramayah, \& Sajilan, 2017). Thereafter, an exploratory factor analysis with the maximum likelihood estimation method and a GEOMIN rotation was performed instruments which showed possible presence of common method bias in order to explore the factor structures of the instruments. Second, 
descriptive statistics were computed to describe the data and determine distribution, and included measuring the means, skewness and kurtosis and standard deviations. Cronbach's alpha was used to determine internal consistency for the instruments. Cut-off values for Cronbach's alpha ( $>0.70$; Nunnally \& Bernstein, 1994) were used to determine scale reliability. Pearson's and Spearman's correlations were used to determine correlations amongst the variables. If the data were non-normally distributed (skewness or kurtosis surpassed +1 or -1 ; Field, 2013), Spearman's correlations were used. Lastly, statistical significance was established at 99\% ( $p \leq 0.01)$, and practical significance was established at 0.30 with a medium effect and 0.50 with a large effect (Steyn \& Swanepoel, 2008).

Third, latent profile analysis (LPA) was used to investigate Objective 1, since it aims to extract patterns in shared variance in order to create meaningful data-driven profiles (Gabriel, Daniels, Diefendorff, \& Greguras, 2015). A stepwise, iterative model comparison process was employed to determine the number of profiles present within the data. First, one latent profile initially specified and with each additional iteration, an extra profile was added until the model failed to converge (Nylund, Asparouhov, \& Muthén, 2007). Second, the latent profiles were examined in relation to model fit indices. Third, once the optimal amount of profiles (relative to the data) was extracted, the latent profile classification categories were investigated to determine if these make theoretical sense. Finally, the relative profile sizes were reported and plotted. Model fit was estimated through the following fit indices: The Lo-Mendell-Rubin likelihood ratio test (LMR) (Lo, Mendell, \& Rubin, 2001) and the significant bootstrap likelihood ratio test (BLRT) were used, assessing the comparative fit between two models that differs by one class. These ratios provide a $p$ value $(<0.01)$ indicating the best fitting model. The lower sample size-adjusted Bayesian information criterion (BIC) was also used as it is a good indicator model fit (Nylund et al., 2007). Lastly, entropy was used to provide an index of model classification quality. Values greater than 0.80 indicated adequate classification quality and values closer to 1.0 indicated better classification quality (Jung \& Wickrama, 2008).

Finally, multivariate analysis of variance (MANOVA) was used to determine differences between the extracted mental health profiles of industrial psychologists and workrole fit, meaningfulness, work engagement and managerial roles (Objective 2 and 3). If the level was 0.05 and less, differences do exist between the three groups (Pallant, 2010). When an effect was significant in MANOVA, a oneway analysis of variance (ANOVA) was used to discover which dependent variables had been impacted. The Bonferroni-type adjustment was implemented to manage inflated type 1 error. The Games-Howell procedure was used to determine whether there were statistical differences between the groups (Pallant, 2010). To measure the effect, partial eta squared $\left(\eta^{2}\right)$ was used. Statistical significance was established at $p<0.01$, with medium effect established at 0.13 and large effect at $0.25(>)$ (Miles \& Shevlin, 2001).

\section{Results}

\section{Common Method Bias and Exploratory Factor Analysis}

To determine whether CMB was present, a Harman's single factor test was conducted. All the observed indicators were entered into an unrotated exploratory factor analysis within Mplus. Model fit for the one-factor model was poor $\left(\square_{(665, N=272)}^{2}=4381.78 ; \quad \mathrm{CFI}=0.56 ; \quad \mathrm{TLI}=0.53\right.$; RMSEA $=0.14 ;$ SRMR $=0.14$ ), and the common shared variance was below the suggested 35\% (Tehseen et al., 2017). Therefore, no single factor could be extracted. However, the results indicated high inter-item correlations between observed variables from the Work-Role Fit and Psychological Meaningfulness Scales. This indicates the probable presence of collinearity, which could inflate the variance in other analyses.

Therefore, another EFA using the maximum likelihood estimation and the GEOMIN (oblique) rotation was conducted to explore the specific factor structures of the WorkRole Fit Scale and the Psychological Meaningfulness Scale. A one-factor model (comprised all ten items) was structurally compared to the original two-factor model (comprised the [4] work-role fit items and the [6] psychological meaningfulness items). Although the two-factor model $\left(\square_{(35 . N=272)}^{2}=126.82 ; \quad\right.$ CFI $=0.97 ; \quad$ TLI $=0.95$; RMSEA $=0.11$; SRMR $=0.02)$ showed slightly better fit than the one-factor model $\left(\square_{(35 . N=272)}^{2}=199.83\right.$; CFI $=$ $0.95 ; \mathrm{TLI}=0.93 ; \mathrm{RMSEA}=0.13 ;$ SRMR $=0.03$ ), the eigenvalues $(>1)$ and scree plot indicated that only one factor is extractable. All the item loadings of the one-factor model were higher than the suggested cut-off $(>0.50$; Wang \& Wang, 2012) and explained $78.33 \%$ of the total variance in the model. In contrast, the two-factor model produced various cross loadings; most of the item loadings for the second factor of the two-factor model were lower than 0.1 and the item loading combinations could not be meaningfully interpreted. As such, a one-factor model was extracted and labelled "Meaningful Work-Role Fit".

\section{Descriptive Statistics}

The descriptive statistics (means, standard deviations, skewness and kurtosis), Cronbach's alpha coefficients as 
well as Pearson's/Spearman's correlations are reported in Table 3. Alpha coefficients on all the scales were higher than 0.70, indicating acceptable internal consistency (Nunnally \& Bernstein, 1994). Furthermore, Spearman's correlations were used to correct for the distribution on the psychological wellbeing component. As can be seen in Table 3, meaningful work-role fit, work engagement, social wellbeing, emotional wellbeing and psychological wellbeing have a statistically and practically significant relationship with one another. Managerial role, in contrast, does not have a statistically and practically significant relationship with meaningful work-role fit, work engagement, social wellbeing, emotional wellbeing and psychological wellbeing.

\section{Latent Profile Analysis}

A latent profile analysis (LPA) with Mplus 8.1 (Muthén \& Muthén, 1998-2018) was carried out on group participants based on their levels of emotional, social and psychological wellbeing. Different models with an increasing number of latent profiles were tested. Significant enhancement from the reference model to the model with more profiles led to a model being retained. Models were compared using the lowest BIC value and entropy values ranging from 0 to 1 , where higher values were better. (Values smaller than 0.60 were not accepted.) The Lo-Mendell-Rubin (LMR LR) test (Lo et al., 2001), the adjusted LMR LR test, as well as the bootstrapped likelihood ratio test (BLRT) (Wang \& Wang, 2012) were used in Mplus to test the number of classes in a mixture analysis. Posterior class membership probabilities, as well as entropy values, were used to specify the quality of class membership.

To determine the LPA model, several steps were followed. The first step included determining the ideal number of latent profiles. Thereafter, an investigation of the latent profile classification was done. The third step included labelling the latent profiles. The prediction of latent profile membership was completed during the fourth step. To determine the number of latent profiles, four models with different numbers of latent profiles were estimated and compared, starting with a single class model and increasing the number of profiles one at a time. The fit indices are reported in Table 4. The Akaike's information criterion (AIC) (1849.62), Bayesian information criterion (BIC) (1871.26) and the sample size adjusted BIC (ABIC) (1852.23) values of the model with one latent class were the largest, indicating that this model has the worst fit.

Next, the quality of the latent profile membership was investigated. The entropy values for the 2-profile and 3 -profile LPA were 0.83 and 0.83 , respectively, indicating a good classification (Clark, 2010). The posterior class membership probabilities for the 3-profile LPA model were 0.92 (profile 1), 0.90 (profile 2) and 0.94 (profile 3); this compares well to the recommended cut-off value of 0.70 or higher (Nagin, 1999).

Thirdly, classes were labelled based on their means for emotional wellbeing, psychological wellbeing and social wellbeing. Profile 1 (languishers) had the highest mean score and comprised 31 (11.4\%) of the participants. Profile 2 (moderately mentally healthy) obtained average scores and comprised 104 (38.4\%) of the sample. Profile 3 (flourishing) obtained high mean scores and comprised 136 $(50.2 \%)$ of the sample. The three latent profiles are illustrated in Fig. 1.

\section{Multivariate Analysis of Variance (MANOVA)}

To test Objectives 2 and 3, a multivariate analysis of variance (MANOVA) was used to determine the difference between meaningful work-role fit, work engagement and managerial role and the three mental health profiles (languishing, moderate mental health and flourishing) of South African industrial psychologists. The mental health profiles were first analysed for statistical significance using Wilk's Lambda statistics. The results of the comparison are presented in Table 5.

Table 3 Descriptive statistics, alpha coefficients and Pearson's/Spearman's correlations

\begin{tabular}{|c|c|c|c|c|c|c|c|c|c|c|}
\hline Item & Mean & SD & Skewness & Kurtosis & $\alpha$ & 1 & 2 & 3 & 4 & 5 \\
\hline Meaningful work-role fit & 3.86 & 0.95 & -.89 & 0.21 & 0.83 & - & - & - & - & - \\
\hline Work engagement & 3.87 & 0.71 & -.84 & 0.93 & 0.82 & $0.65^{*++}$ & - & - & - & - \\
\hline Social wellbeing & 13.62 & 5.49 & -.17 & -.47 & 0.85 & $0.35^{*+}$ & $0.40^{*+}$ & - & - & - \\
\hline Emotional wellbeing & 10.84 & 2.99 & -.92 & 0.70 & 0.73 & $0.43^{*+}$ & $0.43^{*+}$ & $0.66^{*++}$ & - & - \\
\hline Psychological wellbeing $r_{\mathrm{s}}$ & 22.64 & 5.05 & -1.04 & 1.52 & 0.76 & $0.37^{*+}$ & $0.39^{*+}$ & $0.70^{*++}$ & $0.71^{*++}$ & - \\
\hline Managerial role & 1.53 & 0.50 & -.12 & -2.00 & & -.06 & -.06 & -.10 & 0.01 & -.1 \\
\hline
\end{tabular}

${ }^{*} p<0.05$ - statistically significant; $+r>0.30$ — practically significant (medium effect); $++r>0.50$ —practically significant (large effect) Note $r_{\mathrm{s}}=$ Spearman correlation used 
Table 4 Model fit information

\begin{tabular}{lllllll}
\hline Model & AIC & BIC & ABIC & LMR LR test $p$-value & $\begin{array}{r}\text { ALMR LR test } p \text {-value } \\
\text { BLRT } \\
p \text {-value }\end{array}$ \\
\hline 1-class LPA & 1849.62 & 1871.26 & 1852.23 & n.a & n.a & 0.001 \\
2-class LPA & 1584.55 & 1620.61 & 1588.90 & 0.000 & 0.134 & 0.000 \\
3-class LPA & 1473.18 & 1523.66 & 1479.27 & 0.126 & 0.154 & 0.000 \\
4-class LPA & 1417.96 & 1482.87 & 1425.80 & 0.147 & & 0.000 \\
\hline
\end{tabular}

Fig. 1 Three latent profiles

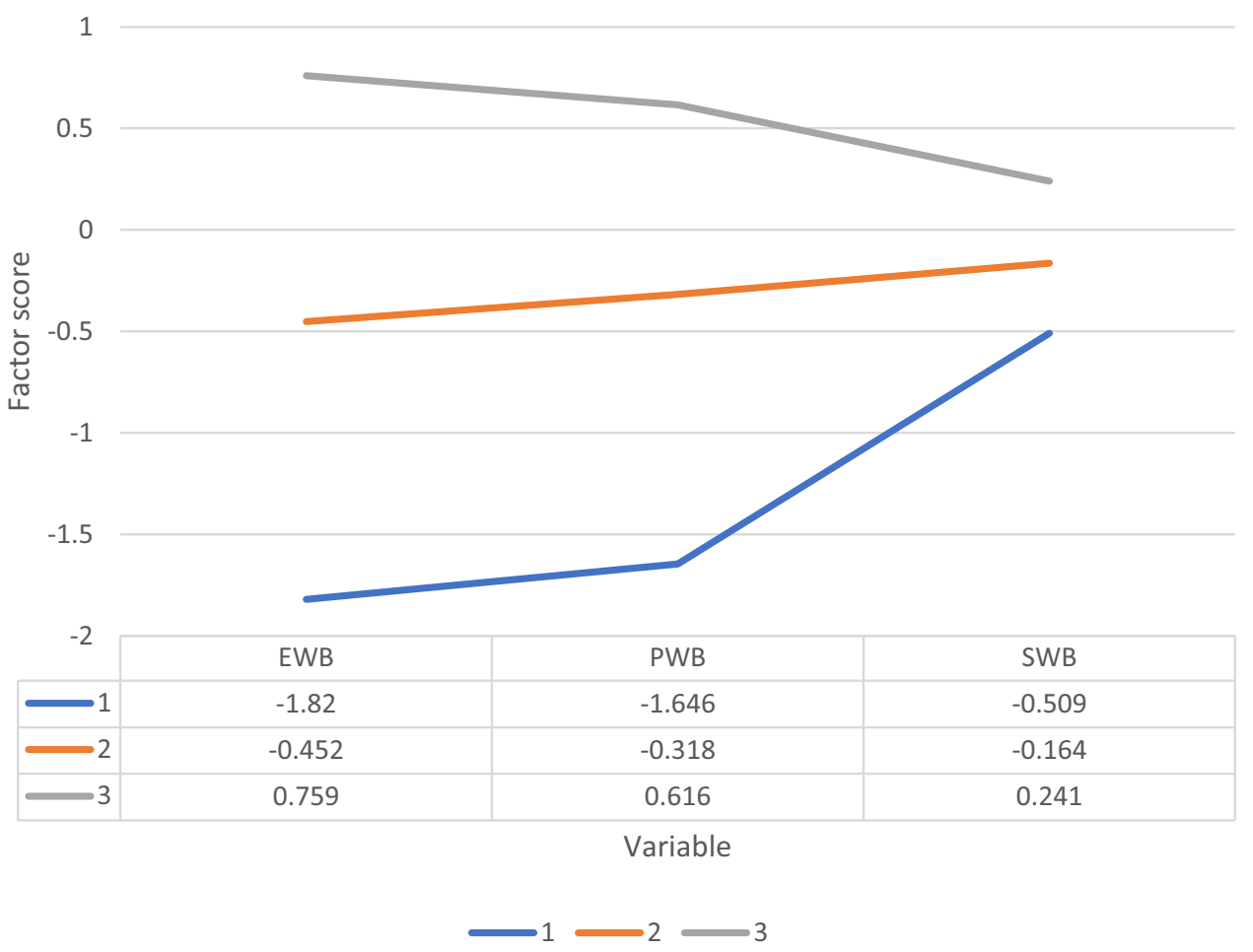

Table 5 MANOVA-differences in meaningful work-role fit, work engagement and managerial role of different mental health profiles of industrial psychologists

\begin{tabular}{llllll}
\hline Variable & Value & $F$ & df & $p$ & Partial eta squared \\
\hline Mental health profiles & 0.67 & 19.33 & 6.00 & $0.00^{*}$ & 0.179 \\
\hline
\end{tabular}

*Statistically significant difference: $p \leq 0.05$

The results showed a statistically significant effect of the different mental health profiles on meaningful work-role fit, work engagement and managerial role $\left(F_{(6,532)}=19.33, p<0.00\right.$, Wilk's $\Lambda=0.67$, partial $\eta^{2}=0.179$ ). This was a medium effect (Cohen, 1988), where $17.9 \%$ of the variance is explained.

Analysis of each individual dependent variable, using Bonferroni adjusted alpha level of 0.0125 , showed that there were significant differences between the levels of meaningful work-role fit $\left(F_{(2.268)}=46.17, p=0.00\right)$, where flourishing showed the highest level of meaningful work-role fit and languishing the lowest. There were also significant differences between the levels of work engagement $\left(F_{(2.268)}=51.33, p=0.00\right)$, where flourishing showed the highest level of engagement and languishing the lowest. There were, however, no significant differences between the levels of managerial roles $\left(F_{(2.268)}=0.33\right.$, $p=0.72$ ).

The results of each individual dependent variable are presented in Table 6. 
Table 6 Differences in meaningful work-role fit, work engagement and managerial role of different mental health profiles of industrial psychologists

\begin{tabular}{llllll}
\hline Variable & Sum of squares & $F$ & df & $p$ & Partial eta squared \\
\hline Meaningful work-role fit & 31.10 & 46.17 & 2 & $0.00^{*}$ & 0.256 \\
Work engagement & 18.73 & 51.33 & 2 & $0.00^{*}$ & 0.277 \\
Managerial role & 0.08 & 0.33 & 2 & 0.72 & 0.002 \\
\hline
\end{tabular}

*Statistically significant difference: $p \leq 0.05$

The significant differences in levels of meaningful work-role fit and work engagement were therefore analysed separately by using ANOVA. The results of ANOVA, based on work-role fit, work engagement and managerial roles, are presented in Table 7.

Results of the ANOVA analysis showed that there were significant differences between both the levels of meaningful work-role fit $\left(F_{(2.268)}=46.17 ; p<0.05\right.$; partial $\left.\eta^{2}=0.26\right)$ and work engagement $\left(F_{(2,} 268\right)=51.33$; $p<0.05$; partial $\left.\eta^{2}=0.28\right)$ on the different mental health profiles.

A post hoc test with a Bonferoni correction provided further information on the mean differences between the three mental health profiles and meaningful work-role fit and work engagement. The results showed that a significant difference in meaningful work-role fit was apparent between languishing and flourishing $(\Delta \overline{\mathrm{x}}=1.24 ; p<0.01)$ and between moderate mental health and flourishing $(\Delta \overline{\mathrm{x}}=0.84 ; p<0.01)$. No significant difference in meaningful work-role fit was found between languishing and moderate mental health $(p>0.01)$. This result implies a significant difference in the experience of meaningful work-role fit from languishing to flourishing and moderate mental health to flourishing, but not from languishing to moderate mental health.

Further, a significant difference in work engagement was apparent between languishing and moderate mental health $(\Delta \overline{\mathrm{x}}=0.44 ; p<0.01)$, between moderate mental health and flourishing $(\Delta \overline{\mathrm{x}}=0.60 ; p<0.01)$, and between languishing and flourishing $(\Delta \overline{\mathrm{x}}=1.04 ; p<0.01)$. This result implies a significant difference in the experience of work engagement from languishing to moderate mental health, to flourishing.

\section{Discussion}

The purpose of this study was to identify distinctive mental health profiles for industrial psychologists, based on Keyes' (2002) mental health framework, and to determine how these profiles differ in respect of work-role fit, psychological meaningfulness, work engagement, and managerial and specialist roles. Given collinearity between work-role fit and psychological meaningfulness, a new factor was created called meaningful work-role fit and used for further exploration. The results indicated that three profiles of mental health for industrial psychologists could be extracted, namely languishing, moderately mentally healthy and flourishing. The results further indicated that global difference between the three mental health profiles and meaningful work-role fit as well as work engagement could be established. However, no significant differences could be found between those who languished and those who were moderately mentally healthy with regards to meaningful work-role fit. Lastly, the results showed that no significant differences between the three mental health profiles and managerial and specialist roles could be established.

\section{The Mental Health Profiles of Industrial Psychologists}

The first objective of this study was to determine the mental health profiles prevalent for industrial psychologists within South Africa. Inline with the findings of Keyes (2002), the results showed that three mental health profiles could be extracted from the data. South African industrial psychologists' mental health could be classified as

Table 7 ANOVA analysis results of meaningful work-role fit, work engagement and managerial role

\begin{tabular}{llllll}
\hline Variable & Flourishers & Moderate mental health & Languishers & $p$ & Partial eta squared \\
\hline Meaningful work-role fit & 4.32 & 3.48 & 3.08 & $0.00^{*}$ & 0.26 \\
Work engagement & 4.21 & 3.61 & 3.17 & $0.00^{*}$ & 0.28 \\
Managerial role & 1.52 & 1.53 & 1.60 & 0.72 & 0.002 \\
\hline
\end{tabular}

* Statistically significant difference: $p \leq 0.05$ 
languishers (11.4\%), moderately mentally healthy (38.4\%) and flourishers $(50.2 \%)$. This implies that more than half of the industrial psychologists experience high levels of emotional, psychological and social wellbeing. This, however, contrasts the findings of Keyes (2002) and Seligman (2012) who argued that only $20 \%$ of the world's population is flourishing (i.e. functioning in the optimal range of mental health). Although there are limitations in this study pertaining to possible self-selection, industrial psychologists that responded may have the necessary skills, abilities and proverbial tools available to manage their work-related demands and stressors. During their academic training, a significant amount of attention is placed on developing the positive psychological capacities (e.g. resilience) of industrial psychology students, in order to help them cope work-related demands (Jorgensen, van Zyl, \& Stander, 2016). This may be an explanation as to why the majority of industrial psychologists within this sample showed higher of flourishing compared to the general population.

\section{Meaningful Work-Role Fit Differs For Languishers and Flourishers}

Further, the results showed that South African industrial psychologists experienced above-average levels of meaningful work-role fit; however, these levels differ in respect of both those who reported to be languishing and flourishing. However, no differences in work-role fit were found between those who reported to be moderately mentally health and the other profiles. This indicates that industrial psychologist within this sample that is languishing experience lower levels of meaningful work-role fit than their flourishing counterparts. Industrial psychologists that are languishing may not feel as though there is a meaningful fit between their work-related roles and their strengths or work-related preferences (Van Zyl et al., 2010; Vogt et al., 2013); in contrast flourishers may experience a greater fit between these two domains. Industrial psychologists that engage in activities (such as HR practices and administration work) which are outside of their personal preferences that are misaligned to their strengths and that do not contribute to something meaningful could influence their mental health (McCann et al., 2013; Rothmann \& Cilliers, 2007).

\section{Work Engagement Differs for Languishers, Moderately Mentally Health and Flourishers}

Differences were found in how work engagement is experienced by industrial psychologists with different mental health profiles. Flourishing individuals experienced significantly higher levels of work engagement than their moderately mentally healthy and languishing counterparts. Industrial psychologists that were languishing showed to experience lower levels of work engagement than those with moderate levels of mental health. This implies that languishers may be more inclined to be emotionally, cognitively and physically disengaged at work (Van Zyl et al., 2010). Industrial psychologists who are flourishing may be inclined to lose themselves in their work-roles and be inclined to experience a sense of flow (Olivier \& Rothmann, 2007). Those whom are moderately mentally healthy, on the other hand may fluctuate between states of engagement and disengagement depending on the environmental or contextual factors in which they are functioning.

\section{Managers and Specialists Did Not Differ in Respect of the Three Mental Health Profiles}

Finally, it was found that no differences on whether an industrial psychologist was in a managerial or a specialist role and their level of mental health could be found. This implies that industrial psychologists with different mental health profiles would not differ according to the managerial roles they occupy. Therefore, it may be that these roles do not affect their mental health.

\section{Limitations and Recommendations}

This study is not without its limitations. First, the crosssectional nature of the design does not allow for meaningful causal assumptions to be inferred relating to the results. At best, this study shows that there are differences in how meaningful work-role fit and work engagement is experienced by individuals that participated in this study; at the time of assessment. Secondly, a census-based sample was employed; however, only a $10 \%$ response rate was obtained. This implies that although the entire population had an equal chance to participate in the study, only a select few contributed. As such, the high level of flourishing in the sample may be an over-estimation of the actual experience of the population. Further, the majority of the participants were "newly registered" industrial psychologists (between 0 and 5 years) which could have skewed the results in a given direction. It is known that individuals within different career stages experience workrelated wellbeing differently, and this specifically was not accounted for in the current study. Further, the use of LPA also has various limitations which need to be considered. First, it employs a data-driven approach to determine subgroups. Careful consideration needs to be given when interpreting profiles as these need to make theoretical sense. Secondly, no cross-validation of the profiles derived from this sample was conducted. It is suggested that future 
research cross-validate the profiles against other measures of mental health. Finally, it is important to remember that essentially all statistical models are false, but some are useful. Given this, it is important to note that different permutations of mental health may exist within the broader population, as our current sample is not fully representative, the results are only applicable to those who participated.

Various recommendations for future research can be made based on the results of this study. The relation between mental health profiles and meaningful work-role fit, work engagement and managerial and specialist roles should be further investigated. Future studies could focus on other antecedents that might influence the mental health of industrial psychologists. Future studies could also focus on how to intervene appropriately with different mental health profiles experienced by industrial psychologists. A larger sample and a longitudinal design could shed more light on the relationships studied. It would also be interesting to compare individuals from different age groups (generations) and gender (male-female) in future studies. Lastly, future research could be conducted in an international context to generalise results, since this study is only focused on the South African context.

\section{Conclusion}

Three distinctive mental health profiles were identified for industrial psychologists in this study, based on Keyes' (2002) dual-continua model, namely flourishing, moderate mental health and languishing. Statistically significant differences existed between the three mental health profiles and meaningful work-role fit and work engagement. The present study yielded important results that could be beneficial for organisations to consider when exploring the mental health of industrial psychologists.

Open Access This article is distributed under the terms of the Creative Commons Attribution 4.0 International License ( http://creativecommons.org/licenses/by/4.0/), which permits unrestricted use, distribution, and reproduction in any medium, provided you give appropriate credit to the original author(s) and the source, provide a link to the Creative Commons license, and indicate if changes were made.

\section{References}

Aguinis, H., Gottfredson, R. K., \& Wright, T. A. (2011). Best-practice recommendations for estimating interaction effects using metaanalysis. Journal of Organizational Behavior, 32(8), 1033-1043.

Ariza-Montes, A., Molina-Sánchez, H., Ramirez-Sobrino, J., \& Giorgi, G. (2018). Work engagement and flourishing at work among nuns: The moderating role of human values. Frontiers in psychology, 9, 1874.
Barkhuizen, H., Jorgensen, L. I., \& Brink, L. (2015). Training the industrial and organisational psychologist as counsellor: Are we doing enough? Acta Commercii, 15(1), 1-12.

Barnard, G., \& Fourie, L. (2007). Exploring the roles and contributions of industrial psychologists in South Africa against a multidimensional conceptual framework (part 2). South African Journal of Industrial Psychology, 33(2), 45-53. Retrieved from https://sajip.co.za/index.php/sajip/article/viewPDFInterstitial/ 376/369.

Benjamin, J., \& Louw-Potgieter, J. (2008). Professional work and actual work: The case of I/O psychologists in South Africa. South African Journal of Psychology, 38(1), 116-135. https://doi.org/10.1177/008124630803800107.

Berlin, K. S., Williams, N. A., \& Parra, G. R. (2014). An introduction to latent variable mixture modeling (part 1): Overview and crosssectional latent class and latent profile analyses. Journal of Pediatric Psychology, 39(2), 174-187.

Charan, R., Drotter, S., \& Noel, J. (2010). The leadership pipeline: How to build the leadership powered company (Vol. 391). Hoboken: Wiley.

Clark, S. L. (2010). Mixture modeling with behavioral dat. (Doctoral dissertation, University of California, Calif.). Retrieved from https://search.proquest.com/openview/ 8a3795d0401e0972f27cb4b4d2c5a5f9/1?pqorigsite $=$ gscholar $\& \mathrm{cbl}=18750 \&$ diss $=\mathrm{y}$.

Cohen, J. (1988). Statistical power analysis for the behavioral sciences (2nd ed.). Hillsdale: Lawrence Erlbaum Associates.

Coetzee, M., \& Van Zyl, L. E. (2014). A review of a decade's scholarly publications (2004-2013) in the South African Journal of Industrial Psychology. SA Journal of Industrial Psychology, 40(1), 1-16.

Cushway, D., \& Tyler, P. (1996). Stress in clinical psychologists. International Journal of Social Psychiatry, 42(2), 141-149. https://doi.org/10.1177/002076409604200208.

De Jager-van Straaten, A., Jorgensen, L., Hill, C., \& Nel, J. A. (2016). Personal growth initiative among industrial psychology students in a higher education institution in South Africa. South African Journal of Industrial Psychology, 42(1), 1-11. https://doi.org/ 10.4102/sajip.v42i1.1283.

Di Benedetto, M., \& Swadling, M. (2014). Burnout in Australian psychologists: Correlations with work-setting, mindfulness and self-care behaviours. Psychology, Health \& Medicine, 19(6), 705-715. https://doi.org/10.1080/13548506.2013.861602.

Drotter, S. (2011). The performance pipeline: Getting the right performance at every level of leadership. Hoboken: Wiley.

Field, A. (2013). Discovering statistics using IBM SPSS statistics (4th ed.). London: Sage Publications.

Field, E., Callaghan, N., Walsh, H., Lord, E., Hudson, M. E., Jones, S., et al. (2018). The role of specialist nurses within a paediatric inherited cardiovascular disease service. Heart, 104, 1-16. https://doi.org/10.1136/heartjnl-2017-BCCA.20.

Figley, C. R. (2002). Compassion fatigue: Psychotherapists' chronic lack of self-care. Journal of Clinical Psychology, 58(11), 1433-1441. https://doi.org/10.1002/jclp.10090.

Gabriel, A. S., Daniels, M. A., Diefendorff, J. M., \& Greguras, G. J. (2015). Emotional labor actors: A latent profile analysis of emotional labor strategies. Journal of Applied Psychology, 100(3), 863-879. https://doi.org/10.1037/a0037408.

Geiser, C. (2012). Data analysis with Mplus. New York: Guilford.

Glazer, S., Kozusznik, M. W., Meyers, J. H., \& Ganai, O. (2014). Meaningfulness as a resource to mitigate work stress. Contemporary Occupational Health Psychology: Global Perspectives on Research and Practice, 3, 114-130. https://doi.org/10.1002/ 9781118713860.ch8.

Grand, J. A., Rogelberg, S. G., Allen, T. D., Landis, R. S., Reynolds, D. H., Scott, J. C., et al. (2018). A systems-based approach to 
fostering robust science in industrial-organizational psychology. Industrial and Organizational Psychology, 11(1), 4-42.

Harman, H. H. (1976). Modern factor analysis. Chicago: University of Chicago press.

Hitge, E., \& Van Schalkwyk, I. (2018). Exploring a group of South African psychologists' well-being: Competencies and contests. South African Journal of Psychology, 48(4), 553-566.

HPCSA. (2011). Regulations defining the scope of the profession of psychology. Retrieved December 1, 2018 from the Health Professions Council of South Africa http://www.hpcsa.co.za.

Jorgensen, L. I., \& Van Zyl, L. E. (In press). Inspiring growth: A framework for training industrial psychology practitioners as counsellors. In L. E. van Zyl \& S. Rothmann (Eds.), Theoretical approaches to multi-cultural positive psychological interventions. NY: Springer.

Jorgensen-Graupner, L. I., \& Van Zyl, L. E. (2019). Inspiring Growth: A Counselling Framework for Industrial Psychology Practitioners. In Positive Psychological Intervention Design and Protocols for Multi-Cultural Contexts (pp. 381-404). Cham: Springer.

Jorgensen, L. I., van Zyl, L. E., \& Stander, M. W. (2016). Training emerging psychologists as multi-cultural contextual coaches. In Coaching Psychology: Meta-theoretical perspectives and applications in multicultural contexts (pp. 357-392). Cham: Springer.

Jung, T., \& Wickrama, K. A. S. (2008). An introduction to latent class growth analysis and growth mixture modeling. Social and Personality Psychology Compass, 2(1), 302-317. https://doi.org/10.1111/j.1751-9004.2007.00054.x.

Kahn, W. A. (1990). Psychological conditions of personal engagement and disengagement at work. The Academy of Management Journal, 33(4), 692-724. https://doi.org/10.5465/256287.

Keyes, C. L. (2002). The mental health continuum: From languishing to flourishing in life. Journal of Health and Social Behavior, 43(2), 207-222. https://doi.org/10.2307/3090197.

Keyes, C. L. (2005). Mental illness and/or mental health: Investigating axioms of the complete state model of health. Journal of Consulting and Clinical Psychology, 73(3), 539-548. https://doi.org/10.1037/0022-006X.73.3.539.

Keyes, C. L. (2007). Journal of Public Mental Health, 6(2), 4-7. https://doi.org/10.1108/17465729200700009.

Khamisa, N., Peltzer, K., Ilic, D., \& Oldenburg, B. (2016). Workrelated stress, burnout, job satisfaction and general health of nurses: A follow-up study. International Journal of Nursing Practice, 22(6), 538-545. https://doi.org/10.1111/ijn.12455.

Khamisa, N., Peltzer, K., Ilic, D., \& Oldenburg, B. (2017). Effect of personal and work stress on burnout, job satisfaction and general health of hospital nurses in South Africa. Health SA Gesondheid, 22(1), 252-258. https://doi.org/10.4102/hsag.v22i0.1011.

Landy, F. J., \& Conte, J. M. (2016). Work in the 21 st century, binder ready version: An introduction to industrial and organizational psychology. Hoboken: Wiley.

Lim, N., Kim, E. K., Kim, H., Yang, E., \& Lee, S. M. (2010). Individual and work-related factors influencing burnout of mental health professionals: A meta-analysis. Journal of Employment Counselling, 47(2), 86-96. https://doi.org/10.1002/ j.2161-1920.2010tb0093.x.

Lo, Y., Mendell, N. R., \& Rubin, D. B. (2001). Testing the number of components in a normal mixture. Biometrika, 88(3), 767-778. https://doi.org/10.1093/biomet/88.3.767.

Lomas, T., Medina, J. C., Ivtzan, I., Rupprecht, S., \& Eiroa-Orosa, F. J. (2018). A systematic review and meta-analysis of the impact of mindfulness-based interventions on the well-being of healthcare professionals. Mindfulness, pp. 1-24.

Marsh, H. W., Lüdtke, O., Trautwein, U., \& Morin, A. J. (2009). Classical latent profile analysis of academic self-concept dimensions: Synergy of person- and variable-centered approaches to theoretical models of self-concept. Structural Equation Modeling, 16, 191-225.

Masyn, K. E. (2013). Latent class analysis and finite mixture modeling. The Oxford Handbook of Quantitative Methods in Psychology, 2, 551-611.

May, D., Gilson, R., \& Harter, L. (2004). The psychological conditions of meaningfulness, safety and availability and the engagement of the human spirit at work. Journal of Occupational and Organizational Psychology, 77, 11-37. https:// doi.org/10.1348/096317904322915892.

McCann, C. M., Beddoe, E., McCormick, K., Huggard, P., Kedge, S., Adamson, C., et al. (2013). Resilience in the health professions: A review of recent literature. International Journal of Wellbeing, 3(1), 60-81. https://doi.org/10.5502/ijw.v3i1.4.

Meyer, J. P., Stanley, L. J., \& Vandenberg, R. J. (2013). A personcentered approach to the study of commitment. Human Resource Management Review, 23(2), 190-202.

Meyers, C. (2007). Industrial psychology. New York: Garnsey Press.

Miles, J., \& Shevlin, M. (2001). Applying regression and correlation: A guide for students and researchers. London: Sage Publications.

Molenberghs, G. (2010). Survey methods and sampling techniques. Interuniversity Institute for Biostatistics and Statistical Bioinformatics (I-BioStat). Retrieved from https://soc.kuleuven.be/ceso/ historischedemografie/resources/pdf/ML/survey10.pdf.

Muthén, L. K., \& Muthén, B. O. (1998-2018). Mplus user's guide (8th ed.). Los Angeles, CA: Muthén \& Muthén. Retrieved from https://www.statmodel.com/download/userguide/MplusUserGuide Ver.8.pdf.

Nagin, D. S. (1999). Analyzing developmental trajectories: A semiparametric, group-based approach. Psychological Methods, 4(2), 139-157. Retrieved from https://pdfs.semanticscholar.org/9c42/ 040f9ce675175ab89d777aa0b263a4f6d0b7.pdf.

Nunnally, J. C., \& Bernstein, I. H. (1994). Psychometric theory (3rd ed.). New York.

Nylund, K. L., Asparouhov, T., \& Muthén, B. O. (2007). Deciding on the number of classes in latent class analysis and growth mixture modeling: A Monte Carlo simulation study. Structural Equation Modeling, 14(4), 535-569. https://doi.org/10.1080/10705510 701575396.

Oberski, D. (2016). Mixture models: Latent profile and latent class analysis. In J. Robertson \& M. Kaptein (Eds.), Modern statistical methods for HCI (pp. 275-287). Cham: Springer.

Ogińska-Bulik, N. (2006). Occupational stress and its consequences in healthcare professionals: The role of type D personality. International Journal of Occupational Medicine and Environmental Health, 19(2), 113-122. https://doi.org/10.2478/v10001006-0016-7.

Olivier, A. L., \& Rothmann, S. (2007). Antecedents of work engagement in a multinational oil company. South African Journal of Industrial Psychology, 33(3), 49-56. Retrieved from http://www.ianrothmann.com/pub/engagement_olivier.pdf.

Pallant, J. (2010). SPSS survival manual (4th ed.). New York: McGraw-Hill.

Peterson, C., \& Seligman, M. E. (2004). Character strengths and virtues: A handbook and classification. New York: Oxford University Press.

Petrou, P., Van den Heuvel, M., \& Schaufeli, W. (2017). The joint effects of promotion and prevention focus on performance, exhaustion and sickness absence among managers and nonmanagers. Personnel Review, 46(8), 1493-1507. https://doi.org/ 10.1108/PR-12-2015-0309.

Rothmann, S., \& Cilliers, F. V. N. (2007). Present challenges and some critical issues for research in industrial/organisational psychology in South Africa. South African Journal of Industrial Psychology, 33(1), 8-17. https://doi.org/10.4102/sajip.v33i1.262. 
Rothmann, S., \& Rothmann, S., Jr. (2010). Factors associated with employee engagement in South Africa. South African Journal of Industrial Psychology, 36(2), 1-12. https://doi.org/10.4102/ sajip.v36i2.925.

Ruderman, M. N., \& Ohlott, P. J. (1994). The realities of management promotion. Greensboro: Center for Creative Leadership.

Rothmann, S., Van Zyl, L. E., \& Rautenbach, C. (2019). Measuring Flourishing@Work Interventions: The Development and Validation of the Flourishing-at-Work Scale. In Positive Psychological Intervention Design and Protocols for Multi-Cultural Contexts (pp. 241-276). Cham: Springer.

Schaerer, M. (2011). Affirming psychology in industrial psychology. (Unpublished master's dissertation). University of Johannesburg, Johannesburg, South Africa.

Seligman, M. E. (2008). Positive health. Applied Psychology, 57(1), 3-18. https://doi.org/10.1111/j.1464-0597.2008.00351.x.

Seligman, M. E. (2012). Flourish: A visionary new understanding of happiness and well-being. New York: Simon and Schuster.

SPSS Incorporated. (2018). SPSS 26.0 for Windows. Chicago: SPSS Inc.

Stanley, L., Kellermanns, F. W., \& Zellweger, T. M. (2017). Latent profile analysis: Understanding family firm profiles. Family Business Review, 30(1), 84-102.

Steyn, H. S., \& Swanepoel, C. J. (2008). Praktiese statistiek (2nd ed.). Potchefstroom: North-West University.

Tehseen, S., Ramayah, T., \& Sajilan, S. (2017). Testing and controlling for common method variance: A review of available methods. Journal of Management Sciences, 4(2), 142-168.

Van Zyl, L. E., Deacon, E., \& Rothmann, S. (2010). Towards happiness: Experiences of work-role fit, meaningfulness and work engagement of industrial/organisational psychologists in South Africa. South African Journal of Industrial Psychology, 36(1), 1-10. https://doi.org/10.4102/sajip.v40i1.1098.

Van Zyl, L. E., Nel, E., Stander, M. W., \& Rothmann, S. (2016). Conceptualising the professional identity of industrial or organisational psychologists within the South African context. South African Journal of Industrial Psychology, 42(1), 13-26. https://doi.org/10.4102/sajip.v42i1.1379.

Veage, S., Ciarrochi, J., Deane, F. P., Andresen, R., Oades, L. G., \& Crowe, T. P. (2014). Value congruence, importance and success and in the workplace: Links with wellbeing and burnout amongst mental health practitioners. Journal of Contextual Behavioral Science, 3(4), 258-264. https://doi.org/10.1016/j.jcbs. 2014.06.004.

Vogt, K., Jenny, G. J., \& Bauer, G. F. (2013). Comprehensibility, manageability and meaningfulness at work: Construct validity of a scale measuring work-related sense of coherence. South African Journal of Industrial Psychology, 39(1), 1-8. https://doi.org/10.4102/sajip.v39i1.1111.

Wang, J., \& Wang, X. (2012). Structural equation modeling: Applications using Mplus. Chichester: Wiley Publishers.

World Health Organisation. (2014, August 15). Mental health: A state of well-being. Retrieved April 3, 2019, from https:// www.who.int/features/factfiles/mental_health/en/.

Publisher's Note Springer Nature remains neutral with regard to jurisdictional claims in published maps and institutional affiliations. 